http://rev.med.panacea.unica.edu.pe

Rev méd panacea. 2014; 4(1): 13-16.

Recibido: 22 de Febrero del 2014 | Aceptado: 03 de Marzo del 2014 | Publicado: 30 de Abril del 2014

CONFICTO DE INTERES: NO DECLARADOS

ARTÍCULO ORIGINAL

\title{
Factores de riesgo asociados a la ruptura prematura de membranas en pacientes atendidas en el Hospital San Juan de Dios de Pisco, 2012
}

\author{
Jorge Ybaseta-Medina ${ }^{1,2, a}$, Mirko Carlos Barranca-Pillman ${ }^{1, b}$, Liliana Ruth Fernández-Enciso ${ }^{1, b}$, Fabiola Elizabeth Vasquez-Lavarello 1,b
}

\author{
Facultad de Medicina, Universidad Nacional San Luis Gonzaga. Ica, Perú ${ }^{1}$ \\ Hospital Santa María del Socorro. Ica, Perú ${ }^{2}$ \\ Medico especialista en ginecologia y obstetriciaa , médico cirujano ${ }^{b}$
}

\begin{abstract}
O bjetivo: Determinar los factores de riesgo asociados a la ruptura prematura de membranas (RPM) en pacientes atendidas en el Hospital San Juan de Dios de Pisco durante el año 2012. Materiales y Métodos: Se realizó un estudio de casos y controles no pareado. La población estuvo conformada por las gestantes atendidas en el Hospital San Juan de Dios de Pisco en el año 2012. Se atendieron 1965 ges tantes, de los cuales 96 fueron diagnosticados como RPM mayor a 22 semanas de gestación, siendo estos los casos y los controles el doble de los casos 192. Para la selección de controles se utilizó el tipo de muestreo aleatorio simple. Para el análisis de los datos se calcularon los Odds Ratio (OR) para identificar los factores de riesgo. Para el cálculo del OR se usó un intervalo de confianza al 95\% de confiabilidad y nivel de significancia $\mathrm{p}<0,05$.Resultados: Los factores de riesgo relacionados al RPM según el grado de asociación son: Infección vaginal $(\mathrm{OR}=13)$; obesidad $(\mathrm{OR}=3)$; infección del tracto urinario $(\mathrm{OR}=2,56)$; gran multípara $(\mathrm{OR}=2,10)$; Hemoglobina entre 9,9-7,1 $\mathrm{grs} / \mathrm{dl}(\mathrm{OR}=2)$ y presentado una asociación más débil con el RPM el grupo de las nulíparas con un $O R=1,9$. Conclusiones: Existen factores de riesgo asociados al RPM que pueden ser modificables mediante un control prenatal estandarizado.
\end{abstract}

Palabras clave: Rotura prematura de membranas fetales; factores de riesgo, embarazo (fuente: DeCS BIREME).

Autor corresponsal: Jorge Ybaseta Medina. Dirección: Calle Fermin Tanguis J-152. Ica, Perú. Teléfono: (51) 956747971. Correo electrónico: jorgeybaseta@yahoo.es

\section{INTRODUCCIÓN}

La ruptura prematura de membranas (RPM) nas entre las 34 y 37 semanas; RPMpt lejos es un accidente obstétrico en el que se pre- del término, que son aquel grupo de embasenta solución de continuidad de las mem- razadas que presentan ruptura de membrabranas corioamnioticas y pérdida de líquido nas con edad gestacional entre las 24 y 34 amniótico después de las 22 semanas de semanas y RPMpt pre viable, cuando la rupedad gestacional y una hora antes del inicio tura ocurre antes de las 24 semanas o antes del trabajo de parto(1), su frecuencia aproxi- del límite de viabilidad, que puede variar madamente es del 10\% de todos los embara- según la institución u hospital donde se lazos, alcanza el $80 \%$ en embarazos a término bore(3).

y en un $20 \%$ de embarazos pre término, siendo responsable de un $30-40 \%$ de los partos prematuros(2).

Cuando la RPM ocurre antes de la semana 37 es conocida como ruptura prematura de membranas fetales pretérmino (RPMpt) (1). Estas pacientes con RPMpt se pueden subdividir según la edad gestacional en tres grupos: RPMpt cerca del término, que son aquellas pacientes con ruptura de membra- como: medio socioeconómico bajo, índice de masa corporal disminuido, sangrado en el segundo y tercer trimestre del embarazo, tabaquismo, deficiencias nutricionales, dilatación cervical, hipercontractilidad, incompetencia cervical, traumática, coito, tactos vaginales repetidos, uso de catéter, amnioscopia, procedimientos de diagnóstico prenatales ,deficiencia de vitaminas, minerales y oligoelementos (Vitamina C, Cobre y Zinc).

También se considera al aumento de la presión intraamniótica, embarazo múltiple, polihidramnios, alteraciones fetales, malformaciones congénitas, anomalías de la presentación (cara, pelviana, hombro, transversa), patologías maternas, abortos previos, hemorragias anteparto, enfermedades del tejido conectivo (síndrome de Ehler-Danlos), conización cervical, sobre distensión uterina por embarazo gemelar o polihidramnios, y antecedente de rotura prematura de membranas.

Cuando la RPMpt se presenta antes de las 34 semanas de edad gestacional, es una etapa crucial, debido a que existe mucha controversia en el manejo pues la prematuridad podría traer consecuencias desastrosas en el neonato.

Entre los factores de riesgo se encuentran: la infección genital por diversos microorganismos (Vaginosis bacteriana, Streptococcus del grupo B, Chlamydia trachomatis, Neisseria gonorrhoeae, Trichomonas vaginalis, etc); siendo el principal factor de riesgo conocido para la presencia de RPM(4).

Además de los procesos infecciosos existen factores de riesgo adicionales implicados en la rotura prematura de membranas, tales
Dentro de las complicaciones neonatales se describen principalmente infección (sepsis neonatal), prematurez, síndrome de dificultad respiratoria y depresión neonatal, que se pueden explicar por aumento en la incidencia de asfixia perinatal por infección fetal, prolapso de cordón, desprendimiento prematuro de placenta, presentaciones distócicas y compresión funicular por oli- 
goamnios.

Este estudio busca identificar los factores de riesgo materno que se asocian a la ruptura prematura de membranas con la finalidad de tratar de orientar a la gestante durante nuestro desempeño como médicos.

\section{MATERIALES Y MÉTODOS}

Se realizó un estudio de casos y controles no pareado. La población estuvo conformada por las gestantes atendidas en el Hospital San Juan de Dios de Pisco en el año 2012. Se atendieron 1965 gestantes, de los cuales 96 fueron diagnosticados como RPM, siendo estos los casos y los controles el doble de los casos (192). Para la selección de controles se utilizará el tipo de muestreo aleatorio simple. Como definición de caso se consideró a todo paciente con diagnóstico de RPM, mayor a 22 semanas de edad gestacional y que cuente con historia clínica completa y como control a todo paciente sin diagnóstico de RPM, mayor a 22 semanas de edad gestacional y que cuente con historia clínica completa. Dentro de los criterios de exclusión consideramos a los pacientes tengan historia clínica incompleta y a pacientes con gestaciones menores a 22 semanas.

Para el desarrollo del estudio, se solicitó al director del Hospital San Juan de Dios de Pisco, el acceso a la información de las historias clínicas del servicio de estadística, además se solicitó a la jefatura del servicio de ginecobstetricia el acceso al sistema informático perinatal (SIP) del Hospital. La información requerida se obtuvo de la base de datos del sistema perinatal y de la revisión de las historias clínicas respectivas. Para la recolección de la información requerida, se utilizó una ficha de recolección de elaboración propia que considera todos los datos requeridos. Toda la información recolectada se transcribirá en formato digital en una hoja de cálculo Microsoft Office Excel $2007^{\mathrm{TM}}$.

\section{RESULTADOS}

Se observa que la mayoría de las madres presentan edades entre 20 y 34 años (77\% de los casos y $80 \%$ de los controles). Evidenciándose además que ningún grupo etario se asocia con la RPM ( $p>0,05)$.

Se puede observar al dividir a los casos en primigestas y multigestas, con sus respectivos controles, no se determinó que estos fueran un factor de riesgo (OR:1,6; IC: 0,9-2,6)

Igualmente al dividir las multigestas, en aquellas con periodo integenesico $(\mathrm{Pl})<2$ años y con $\mathrm{PI}>2$ años no se encontró que estas fueran factores de riesgo para el desarrollo de RPM. (PI<2 años; OR: 0,6; IC: 0,34-1,11/ PI>2años; OR:0,6, IC: 0,6-1,6).

\begin{tabular}{|c|c|c|c|c|c|}
\hline \multirow{2}{*}{$\begin{array}{ll} & \text { TABLA } 1 \\
\text { Factores } & \end{array}$} & \multicolumn{5}{|c|}{$\begin{array}{l}\text { Factores de riesgo asociados a la ruptura prematura de mem- } \\
\text { branas en pacientes atendidas en el Hospital San Juan de Dios } \\
\text { de Pisco, } 2012\end{array}$} \\
\hline & Casos & Controles & O.R. & I.C. $95 \%$ & $\mathbf{P}$ \\
\hline Edad materna & $\mathrm{N}(\%)$ & $\mathrm{N}(\%)$ & & & \\
\hline$\leq$ de 19 años & $16(17)$ & $26(14)$ & 1,2 & $0,6-2,4$ & 0,9 \\
\hline 20-34 años & $74(77)$ & $154(80)$ & 0,9 & $0,6-1,3$ & 0,7 \\
\hline$\geq 34$ años & $6(6)$ & $12(6)$ & 1,00 & $0,3-2,7$ & 1,00 \\
\hline \multicolumn{6}{|l|}{ PI } \\
\hline Primigesta & $44(46)$ & $66(34)$ & 1,3 & $0,8-2,0$ & 0,2 \\
\hline$<2$ años & $18(19)$ & $58(30)$ & 0,6 & $0,3-1,1$ & 0,1 \\
\hline$>2$ años & $34(35)$ & $68(36)$ & 1,0 & $0,6-1,6$ & 1,0 \\
\hline \multicolumn{6}{|l|}{ Paridad } \\
\hline Nulipara (o partos) & $32(33)$ & $44(23)$ & 1,9 & $0,9-2,4$ & $<0,05$ \\
\hline Multipara (1-4 partos) & $62(65)$ & $146(76)$ & 0,8 & $0,5-1,3$ & 0,4 \\
\hline Gran multípara (> 4 partos) & $02(2)$ & $2(1)$ & 2,1 & $0,3-14,4$ & $<0,05$ \\
\hline \multicolumn{6}{|l|}{ Hemoglobina materna } \\
\hline$\geq 11 \mathrm{grs} / \mathrm{dl}$ & $74(77)$ & $158(82)$ & 0,9 & $0,6-1,4$ & 0,065 \\
\hline $10-10,9 \mathrm{grs} / \mathrm{dl}$ & $02(02)$ & $11(6)$ & 1,0 & $0,3-1,2$ & 1,67 \\
\hline $9,9-7,1 \mathrm{grs} / \mathrm{dl}$ & $18(19)$ & $19(10)$ & 2,0 & $0,9-3,4$ & 0,001 \\
\hline$\leq 7 \mathrm{grs} / \mathrm{dl}$ & $02(02)$ & $4(2)$ & 1,0 & $0,1-5,5$ & 1,00 \\
\hline \multicolumn{6}{|l|}{ IMC Materno } \\
\hline Bajo peso $(<19,8)$ & $12(13)$ & $22(11)$ & 1,0 & $0,5-2,9$ & 0,8 \\
\hline Normal $(19,8-26)$ & $54(55)$ & $136(71)$ & 0,8 & $0,5-1,2$ & 0,3 \\
\hline Sobrepeso $(26,1-29)$ & $18(19)$ & $26(14)$ & 1,4 & $0,7-2,6$ & 0,3 \\
\hline Obesidad (>29) & $12(13)$ & $08(04)$ & 3,0 & $1,2-7,5$ & $<0,05$ \\
\hline \multicolumn{6}{|l|}{ Infección vaginal } \\
\hline $\mathrm{Si}$ & $40(41$ & $06(03)$ & 13 & $5,5-32,5$ & $<0,05$ \\
\hline No & $56(59)$ & $186(97)$ & 0,6 & $0,4-0,9$ & $<0,05$ \\
\hline \multicolumn{6}{|l|}{ ITU } \\
\hline Si & $32(34)$ & $25(13)$ & 2,6 & $1,4-4,6$ & $<0,05$ \\
\hline No & $60(66)$ & $167(87)$ & 0,7 & $0,4-1,1$ & 0,09 \\
\hline
\end{tabular}

PI: Periodo intergenesico IMC: Índice de Masa Corporal ITU: Infección del Tracto Urinario

Se puede observar que la mayor parte de los casos se encuentran dentro del grupo de las multíparas (casos=62, 65\%; controles $=146$, $76 \%$ ), aunque estadísticamente no se asocian con la RPM ( $p>0,05)$.

Además se puede apreciar que los grupos de gestantes nulíparas y gran multípara si se encuentran asociados a la RPM $(p<0,05)$, mientras que el grupo de las nulíparas presenta una asociación positiva débil (O.R.=1,91), ser gran multípara se asocia en mayor grado con la RPM $(O . R .=2,10)$.

También se observó que los grupos de casos y controles que presentan hemoglobina 9,9 $7,1 \mathrm{gr} / \mathrm{dl}$ se asocian estadísticamente con la RPM ( $p<0,05, O . R .=2)$. Mientras que los grupos con hemoglobina $\geq 10 \mathrm{gr} / \mathrm{dl} y \leq a \mathrm{gr} / \mathrm{dl}$ no presentan asociación con la RPM ( $>>0,05$, O.R.=1).

A su vez se aprecia que la mayor parte de casos y controles presentan más de $5 \mathrm{CPN}$ (50; $52 \%$ y 90; $47 \%$ respectivamente). Además se puede observar que todos los grupos de gestantes no se encuentran asociados con la RPM ( $p>0,05, O . R .=1)$.

\section{DISCUSIÓN}

La causa de la rotura prematura de membranas es multifactorial, pues comprende complicaciones previas o enfermedades concomitantes (5). Su incidencia varía entre el 1,6 y el $21 \%$ de todos los nacimientos y constituye una de las entidades obstétricas más relacionadas con la morbilidad y mortalidad materno-perinatal, su diagnóstico todavía es fundamentalmente clínico (6).

La incidencia de RPM en el lapso de este estudio encontró una cifra del $5 \%$. A nivel internacional la incidencia varía entre 1,6 y el 21\%, a nivel nacional la frecuencia de RPM se da entre 16 a 21\% según la Guía Técnica del Ministerio de Salud del año 2007. En el Instituto Nacional Materno Perinatal tenemos que en el año 2007 fue $8.37 \%$. En el Hospital Santa Rosa durante el año 2010 se reportó una incidencia de RPM entre 2 a 4\%. Asimismo Yaranga (7) en el año 2003 reporta una incidencia de RPM de 18:3\% en el Hospital Santa María del Socorro de Ica.

Comparando nuestros resultados con los encontrados a nivel nacional e internacional 
se puede observar que nuestro resultado es congruente con diversas bibliografías e incluso nuestro resultado se encuentra en menor porcentaje que los hospitales de Lima e Ica donde su población de estudio es similar.

En la tabla 1 se observa que el mayor grupo poblacional del estudio se encuentra entre 20 y 34 años, sin embargo ningún grupo etario se asocia con la RPM. Resultados similar al trabajo titulado resultados materno perinatales de la RPM pretérmino realizado en Hospital Daniel Alcides Carrión del Callao durante los años 2006 a 2008, mostrando que el grupo etario de 20 a 35 años presentó la mayor cantidad de casos (62\%). Atauje Quispe en su estudio "Complicaciones materno neonatales del manejo activo versus expectante de la Ruptura Prematura de Membranas" realizado en el instituto Nacional materno Perinatal de Lima durante el año 2003 reporta que el 62,2\% se encontraba en edad comprendida entre 20 a 34 años(8). A nivel internacional tenemos a Morgan Ortiz y cols. en México quienes reportaron que no encontraron asociación entre la edad materna y la RPM. Sin embargo hay estudios que difieren con nuestros resultados. Chamy en su estudio "Riesgo obstétrico y perinatal en embarazadas mayores de 35 años" realizado en Chile durante el año 2009, encuentra como factor de riesgo para RPM la edad mayor a 40 años (9).

El grupo etario que mayor número de casos tiene en nuestro estudio se puede explicar porque en la edad entre 20 y 34 años la vida sexual es más activa, lo que da mayor oportunidad a la gestación.

Sobre los resultados del periodo intergenésico, nuestros hallazgos no muestran asociación con la RPM, discordante con la bibliografía revisada (8), que indican un periodo intergenésico corto o muy largo posterior a un embarazo a término, aumenta el riesgo de complicaciones obstétricas así tenemos: en una publicación del Consorcio CATALYST realizado en Latinoamérica durante los años 1985-2000; describio que las mujeres con intervalos intergenésicos menores de 15 meses tuvieron riesgo más alto de presentar RPM, el trabajo realizado por Morgan y cols. concluye que en grupos con periodo intergenésicos menores a 18 meses presentan una mayor asociación con la RPM (8).

El intervalo entre embarazos es importante porque permite a la madre recuperarse después de un parto, sin embargo en nuestro estudio la mayor población de estudio fueron primigestas, esto explicaria la falta de congruencias con otros estudios.

Con respecto a la paridad, nuestros resultados muestran que las gran multíparas son el grupo de gestantes que presentó un mayor grado de asociación con la RPM (O.R.= 2,10) seguidos de las nulíparas $(O . R .=1,90)$ existen estudios que corroboran nuestros resultados, como el de Martínez en Cuba quien público "Análisis de los casos con rotura prematura de membranas", donde concluyó que la multiparidad fue un factor de riesgo para RPM, otro estudio de Martínez Gonzales encuentro que el $48,6 \%$ de los casos eran nulíparas, no obstante Hiertberg y cols. encontraron una elevada frecuencia de nulíparas con rotura de membranas a término (10).

En el mismo trabajo de Martínez se encuentra que la multiparidad no se comporta como factor de riesgo, esta diferencia con nuestros hallazgos puede deberse a que tomamos gestaciones mayores de 34 semanas y Martínez lo hizo en partos mayores a 22 semanas (8).

La anemia en el embarazo se ha relacionado con la falta de aumento del volumen plasmático materno, con el consecuente menor riego sanguíneo y función placentaria inadecuada. Una adecuada expansión del volumen plasmático parece estar relacionada con una disminución de la viscosidad de la sangre para una mejor irrigación placentaria, estas alteraciones hematológicas se han asociado con cinco problemas obstétricos: aborto, rotura prematura de membranas, parto prematuro, oligohidramnios y bajo peso al nacer (13).

Es probable que los grupos que tienen concentraciones de hemoglobina $(\mathrm{Hb})$ menor a 8.9 grs/dl no tengan relación con la RPM, debido quizás a que el número de sus poblaciones son escazas.

Observamos que la mayor población de casos se encuentra en el grupo de índice de masa corporal (IMC) entre 19,8-26 (normal) con el $55 \%$ y que el único grupo que está asociado a la RPM, es el grupo con IMC $>29$ teniendo un $\mathrm{OR}=3$ y $\mathrm{P}<0,05$. Nuestros hallazgos difieren de lo encontrado por Araujo-Anco, quien no hallo asociación entre el IMC y la RPM, sin embargo Kovavisarach en el 2000 en Tailandia encontró que un IMC menor de 20 en una gestante a término se asocia con la RPM (16), pudiendo ser estas diferencias por las características de la población en estudio.

Encontramos asociación entre la presencia de infección del tracto urinario (ITU) y la RPM (O.R. $=2,56$ y $p<0,0014$ ), la RPM es 2,56 veces más frecuente en caso que tuvieron ITU. Concordante con el estudio de Fabián quien hallo que el $37 \%$ de su población presento ITU. Pero es discordante con el estudio de Morgan que no encuentra asociación entre ITU y RPM (18).

Probablemente esta diferencia se deba a la diferencia de poblaciones y que en los estu- dios de Morgan no utilizaron el urocultivo como diagnóstico de ITU sino utilizaron el examen completo de orina.

Se observa que la metrorragia solo representa un $8 \%$ de los casos no asociándose al RPM (O.R. $=0,72$ y $P>0,05)$. Aunque es descrito en la literatura como factor de riesgo para RPM posiblemente porque el coágulo coriodecidual y su subsecuente disolución afecten la integridad bioquímica y la nutrición de las membranas, predisponiéndolas a la RPM (17), esta investigación no es concordante con nuestros resultados probablemente porque el número de casos con metrorragia es muy pequeño.

Podemos concluir que la ruptura prematura de membrana es una complicación usual en la práctica médica, esta puede aumentar la incidencia en la morbilidad y mortalidad materna y fetal. Su diagnóstico todavía es fundamentalmente clínico, la incidencia de RPM en el Hospital San Juan de Dios de Pisco en el año 2012 fue de $5 \%$, entre los factores de riesgo que relacionados al RPM según el grado de asociación fueron la infección vaginal; la obesidad; la infección del tracto urinario; la gran multípararidad; la hemoglobina entre 9,9-7,1 grs/dl y con una asociación más débil con la RPM, la nulíparidad.

\section{REFERENCIAS BIBLIOGRÁFICAS.}

1. Mercer BM. Preterm premature rupture of the membranes. Obstet Gynecol 2003; 101:178-93.

2. Gómez R. Rotura prematura de membranas. Boletín Perinatal Ministerio de salud de Chile 2001; 1(1): 5-23.

3. Vigil de Gracia P, Savransky R, Pérez Wuff J, Delgado Gutiérrez J, Nunez Moráis E. Ruptura prematura de membranas. Guía clínica de la Federación Latino Americana de Sociedades de Ginecología y Obstetricia; Guía № 01.2011.

4. Olivares AS, Pliego Pérez AR. Ensayo clínico del tratamiento de la infección vaginal durante el embarazo y su relación con la incidencia de ruptura prematura de membranas. Rev. Sanidad Militar Mex. 2000; 54:4-8

5. Fred Morgan Ortiz y Cols. Factores sociodemográficos y obstétricos asociados con rotura prematura de membranas. Ginecol Obstet Mex 2008;76(8):468-75.

6. Vázquez Niebla JC, Vázquez Cabrera J, Rodríguez $P$. Epidemiology of the premature rupture of membranes in a gynecological and obstetric hospital. Rev Cubana Obstet Ginecol [revista en la Internet]. 2003 Ago [citado 2014 Feb 13]; 29(2) [Link]

7. Yaranga T, Huamaní Q. Prevalencia y factores asociados a RPM en el Hospital Santa María del Socorro de Ica, 2003. Tema libre II congreso Nacional de Médicos Residentes. 
8. Araujo-Anco C. Estimación del grado de asociación de los factores de riesgo en pacientes con ruptura prematura de membranas, atendidas en el Hospital Hipólito Unanue de Tacna durante el periodo 20062010. (Tesis para optar título Médico Cirujano). Universidad Nacional Jorge Basadre Grohmann-Tacna. pp 105.

9. Morgan Ortiz F, Gómez Soto $Y$, Valenzuela Gonzales I, Gonzales Beltrán A, Quevedo Castro E, Osuna Ramírez I. Factores sociodemográficos y obstétricos asociados con rotura prematura de membranas. Ginecol Obstet Mex 2008;76(8):468-7

10. Martínez González LR, Valladares Hernández M, Villate JL. Análisis de los casos con rotura prematura de membranas y menos de 34 semanas. Rev Cubana Obstet Ginecol [revista en la Internet]. 1998 Dic [citado 2014 Feb 14] ; 24(3): 145-150. [Link].

FINANCIAMIENTO: Autofinanciado

CONTRIBUCIONES DE AUTORÍA: JYM, MCBP, LRFB y FEVL participaron en el diseño del estudio, el análisis de los datos, revisaron críticamente el artículo y aprobaron la versión final.
11. Iglesias Benavides J, Tamez Garza L. Anemia y embarazo, su relación con complicaciones maternas y perinatales. Medicina Universitaria 2009; 11(43):95-98

12. Ferguson SE, Smith GN, Salenieks ME, Windrim R, Walker MC. Preterm Premature Rupture of Membranes: Nutritional and Socioeconomic Factors. Obstet Gynecol 2002; 100: 1250-1256.

13. Steer PJ. Maternal hemoglobin concentration and birth weight. Am J Clin Nutr 2000; 71:1285S-7S.

14. Tipiani O, Tomatis C. El control prenatal y el desenlace maternoperinatal. Rev Per Ginecol Obstet. 2006; 52(4):46-48.

15. World Health Organization. Antenatal Care Randomized Trial. Manual for the implementation of the new model. Geneva: Department of Reproductive Health and Research, Family and Community Health, World Health Organization; 2002.

16. Kovavisarach E, Sermsak P. Risk factors related to premature rupture of membranes in term pregnant women. Aust N Z J Obstet Gynaecol 2000 Feb; 40 (1)30-2

17. León González H, Nitola M. Guía de manejo de Ruptura prematura de membranas. Asociación Bogotana de Obstetricia y Ginecología. [Link]

18. Fabian Velasquez E. Factores de riesgo materno asociados a ruptura prematura de membranas pretérmino en pacientes atendidas en el Instituto Nacional Materno Perinatal durante el periodo enerodiciembre, 2008. Lima: Universidad Nacional Mayor de San Marcos; 2006.

\title{
Risk factors associated with premature rupture of membranes in patients treated at the Hospital San Juan de Dios in Pisco, 2012
}

\begin{abstract}
bjective: To determine the risk factors associated with premature rupture of membranes (PROM) in patients treated at the San Juan de Dios Hospital in Pisco in 2012. Materials and Methods: A casecontrol study was performed unpaired. The population consisted of pregnant women treated at the San Juan de Dios Hospital in Pisco in the year 2012. 1965 pregnant women were attended, of whom 96 were diagnosed as PROM increased to 22 weeks of gestation, which are the cases and controls the double cases 192. in selecting controls the type of simple random sampling was used. For data analysis the odds ratio (OR) were calculated to identify risk factors. To calculate the OR confidence interval $95 \%$ confidence interval and significance level of $\mathrm{p}<0.05$ was used. Results: Risk factors related to PROM depending on the degree of association are: vaginal infection $(O R=13)$; obesity $(O R=3)$; urinary tract infection $(O R=2.56)$; grand multiparity ( $O R=2.10$ ); Hemoglobin between 9.9 to $7.1 \mathrm{~g} / \mathrm{dl}(\mathrm{OR}=2)$ and presented a weaker association with PROM nulliparous group with an $O R=1.9$. Conclusions: There are risk factors associated with the PROM that can be modified using a standardized prenatal care.
\end{abstract}

Keywords: Premature rupture of fetal membranes; risk factors, pregnancy. (source: MeSH NLM)

\section{CITA SUGERIDA.}

Ybaseta-Medina J, Barranca-Pillman MC, Fernández-Enciso LR, Vasquez-Lavarello FE. Factores de riesgo asociados a la ruptura prematura de membranas en pacientes atendidas en el Hospital San Juan de Dios de Pisco, 2012 . Rev méd panacea.2014; 4 (1):13-16. 